\title{
Incidentally discovered COVID-19 pneumonia: the role of diagnostic imaging
}

\author{
Chiara Pozzessere $^{1} \cdot$ David C. Rotzinger ${ }^{2,3}$ (D) Benoit Ghaye ${ }^{4} \cdot$ Frédéric Lamoth $^{5,6} \cdot$ Catherine Beigelman-Aubry $^{2}$
}

Received: 15 April 2020 / Revised: 15 April 2020 / Accepted: 23 April 2020 / Published online: 4 May 2020

(C) European Society of Radiology 2020

Dear Editor,

Numerous healthcare professionals are involved in responding to the worldwide COVID-19 outbreak, and the role of radiologists has been recently emphasized [1]. One of the pointed roles in this setting is the early detection of radiological features suspicious of COVID-19 pneumonia. Currently, the definitive diagnosis of COVID-19 relies on real-time reverse transcription-polymerase chain reaction (RT-PCR) on a nasopharyngeal swab or other respiratory specimens. However, the sensitivity of RT-PCR depends on several factors, including the quality of the sampling, and the viral burden at the time of specimen collection, explaining that some cases may be missed [2]. Conversely, distinct radiological findings on chest CT strongly suggest the diagnosis of COVID-19 pneumonia both in symptomatic and asymptomatic patients [3, 4], even though the sensitivity of imaging remains limited as well, especially in the early phase of the disease.

Interestingly, chest $\mathrm{CT}$ can be abnormal even if there are no clinical signs. Given the current prevalence of the disease, we can expect incidental detection of COVID-19 pneumonia on examinations not directly performed for this reason. This situation is of critical importance since radiologically visible

Electronic supplementary material The online version of this article (https://doi.org/10.1007/s00330-020-06914-6) contains supplementary material, which is available to authorized users.

David C. Rotzinger

david.rotzinger@chuv.ch

1 Department of Radiology, AUSL Toscana Centro San Giuseppe Hospital, Empoli, Italy

2 Department of Diagnostic and Interventional Radiology, Lausanne University Hospital (CHUV) and University of Lausanne (UNIL), Rue du Bugnon 46, 1011 Lausanne, Switzerland

3 Department of Diagnostic and Interventional Radiology, Cardiothoracic and Vascular Imaging Division, Lausanne University Hospital (CHUV), Rue du Bugnon 46, 1011 Lausanne, Switzerland
COVID-19 pneumonia is associated with potential virus transmission.

Figure 1 shows an example of COVID-19 pneumonia incidentally discovered on chest CT. In supplementary material, other cases are provided, including one case of detection during breast MRI (S2-4), all preceding clinical signs and/or microbiological documentation of infection. The CT findings included typical features of COVID-19 pneumonia, such as multiple bilateral areas of ground-glass opacities (GGO) with reticulation or consolidation $[5,6]$. A peripheral and posterior predominance was commonly observed, while in one case (S3), GGO were predominantly located in the central parenchyma. Such findings have been described during the ultraearly asymptomatic stage of the disease, 1-2 weeks after exposure, but could also represent improving abnormalities of previously undetected severe findings in asymptomatic patients [7]. The vascular enlargement sign is an additional finding that can help distinguish COVID-19 from viral pneumonia (S3) $[4,8]$. The cases presented were identified in the early days of the outbreak in Europe. At that time, specific pathways mainly differentiating "COVID" from "non-COVID" patients" were in the process of being implemented in the emergency departments (ED), intensive care units (ICU),

4 Department of Radiology, Université Catholique de Louvain, Cliniques Universitaires Saint-Luc, avenue Hippocrate 10, 1200 Brussels, Belgium

5 Department of Medicine, Infectious Diseases Service, Lausanne University Hospital (CHUV) and University of Lausanne (UNIL), Rue du Bugnon 46, 1011 Lausanne, Switzerland

6 Department of Laboratories, Institute of Microbiology, Lausanne University Hospital (CHUV) and University of Lausanne (UNIL), Rue du Bugnon 46, 1011 Lausanne, Switzerland 


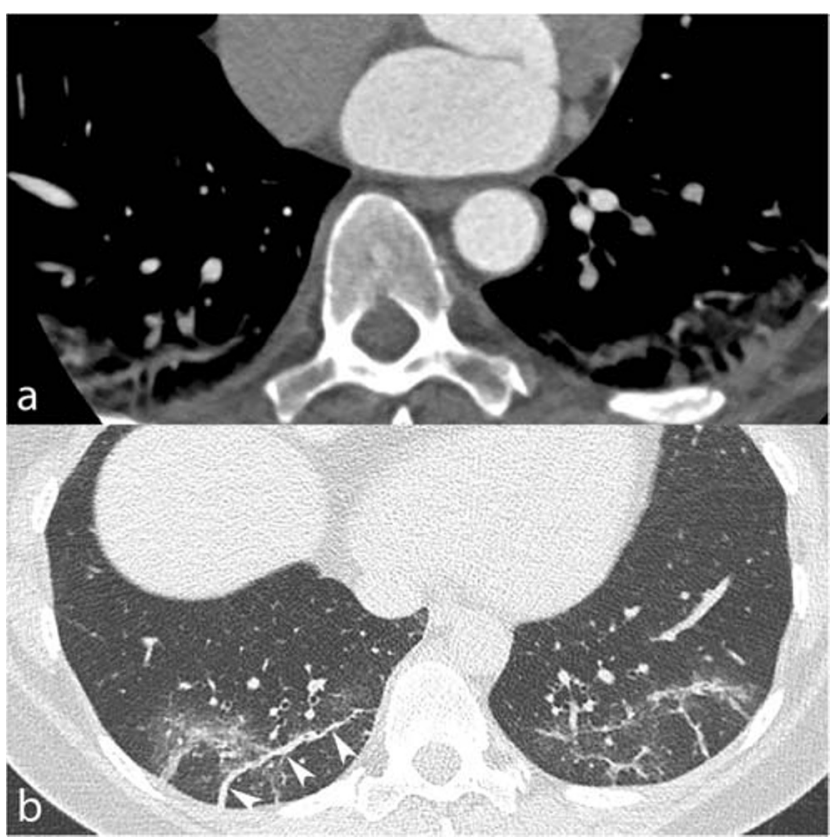

Fig. 1 A 50-year-old female outpatient underwent ECG-gated coronary $\mathrm{CT}$ angiography for exertional dyspnea and repolarization abnormalities at ECG. On the angiographic phase (a), pulmonary abnormalities were found in the dependent areas of both lower lobes by the radiology resident and immediately investigated by a low-dose thoracic $\mathrm{CT}$ in prone position (b). The findings included peripheral ground-glass opacities and fibrous streaks (white arrowheads) in the posterior portion of the lower lobes. While the patient neglected her symptoms in the first place, she admitted a cough, headache for 10 days, and low-grade fever $\left(38^{\circ} \mathrm{C}\right)$ on interrogation. The patient was then sent to the COVID-19 unit, and RT-PCR was positive for SARS-CoV-2

and infectious disease departments [9]; however, such measures were not systematically applied in all hospital areas yet. In this regard, specific management was pursued, including the requirement to wear a mask, immediate information of the suspicious imaging findings to the patient and the referring physician, and activation of the RT-PCR test procedure, as well as careful disinfection of the CT suite. All these infection control measures aimed to prevent potential transmission to other individuals, including other patients who are potentially vulnerable, radiology staff, relatives, and any other personal contacts.

During the COVID-19 outbreak, the radiology staff, especially radiology technicians, have to be aware and trained to any incidental detection of appearances suggestive of SARS$\mathrm{CoV}-2$ infection. Such findings may be discovered not only on chest $\mathrm{CT}$ but, during any imaging performed for other clinical situations, also when the lungs are partially visible in nonpneumological studies. Therefore, while specific tracks for suspected or known COVID-19 patients have been established, the healthcare team should keep in mind that asymptomatic or paucisymptomatic carriers are potentially present in the non-COVID-19 pathway. Several consequences flow from this. First, radiology technicians might be unexpectedly exposed and should be provided with adequate protective equipment. Second, we recommend reviewing all available chest images within any imaging examination as soon as possible before the patient leaves the radiology facility to identify suggestive abnormalities. Third, in case of suggestive findings, the CT suite needs to be appropriately cleaned before the next examination, while patients should be quickly directed to the COVID-19 pathway. Taken together, these actions are likely to slow down the clinical workflow, and prolonged waiting times for outpatients should be avoided.

Of note, once the outbreak will be under control and the number of cases declines, these emergency measures will be progressively discontinued. At the same time, the spread of SARS-CoV-2 will probably not end overnight, and some cases will still be diagnosed in the following months. Maintaining limited but adequate precautionary measures would be warranted to safeguard the health of the community, including healthcare actors and vulnerable persons. In particular, we suggest continuing to review chest images immediately after the acquisition to promptly detect suggestive features of COVID-19 pneumonia, even when the examination is performed for other clinical indications.

Acknowledgments The authors gratefully acknowledge colleagues in the field, who were directly or indirectly involved with patient care: Prof. Reto A. Meuli, Dr. Sahar Ghassem-Zadeh, Dr. Gerogios Sgourdos, Dr. Louise Pichon, Dr. Sandro Santini, and Dr. Giulia Zipoli.

Funding information The authors state that this work has not received any funding.

\section{Compliance with ethical standards}

Guarantor The scientific guarantor of this publication is Catherine Beigelman-Aubry, MD.

Conflict of interest The authors of this manuscript declare no competing relationships.

Statistics and biometry No complex statistical methods were necessary for this paper.

\section{Informed consent Not applicable.}

Ethical approval The local ethics committee did not require ethical review due to the observational nature of the work, involving less than five individuals.

Methodology • Not applicable

\section{References}

1. Kim H (2020) Outbreak of novel coronavirus (COVID-19): what is the role of radiologists? Eur Radiol. https://doi.org/10.1007/s00330$020-06748-2$ 
2. Wang W, Xu Y, Gao R et al (2020) Detection of SARS-CoV-2 in different types of clinical specimens. JAMA. https://doi.org/10.1001/ jama.2020.3786

3. Shi H, Han X, Jiang $\mathrm{N}$ et al (2020) Radiological findings from 81 patients with COVID-19 pneumonia in Wuhan, China: a descriptive study. Lancet Infect Dis 20:425-434

4. Ye Z, Zhang Y, Wang Y, Huang Z, Song B (2020) Chest CT manifestations of new coronavirus disease 2019 (COVID-19): a pictorial review. Eur Radiol. https://doi.org/10.1007/s00330-020-06801-0

5. Pan Y, Guan H (2020) Imaging changes in patients with 2019-nCov. Eur Radiol. https://doi.org/10.1007/s00330-020-06713-z:1-2

6. Zhou Z, Guo D, Li C et al (2020) Coronavirus disease 2019: initial chest CT findings. Eur Radiol. https://doi.org/10.1007/s00330-02006816-7

7. Pan Y, Guan H, Zhou S et al (2020) Initial CT findings and temporal changes in patients with the novel coronavirus pneumonia (2019-
nCoV): a study of 63 patients in Wuhan, China. Eur Radiol. https:// doi.org/10.1007/s00330-020-06731-x

8. Qanadli SD, Beigelman-Aubry C, Rotzinger DC (2020) Vascular changes detected with thoracic CT in coronavirus disease (COVID19) might be significant determinants for accurate diagnosis and optimal patient management. AJR Am J Roentgenol 2020;W1. https://doi.org/10.2214/AJR.20.23185

9. World Health Organization (2020) Operational considerations for case management of COVID-19 in health facility and community: interim guidance. World Health Organization. Available via https:// apps.who.int/iris/handle/10665/331492

Publisher's note Springer Nature remains neutral with regard to jurisdictional claims in published maps and institutional affiliations. 